\title{
Two theorems in general metric space with $\rho$-distance
}

\author{
Salwa Salman Abed, Hiba Adel Jabbar \\ salwaalbundi@yahoo.com \\ hiba.ade185@yahoo.com
}

Dep. Of Math. Colle. Of Education for Pure Sciences Ibn Al-Haitham University of Baghdad

\section{ABSTRACT}

In this paper, we prove two theorems about fixed point and coupled coincidence point in generalized b-metric space via $\rho$-distance for a mapping satisfying a contraction condition.

\section{Keywords}

Weak Contractions; fixed Points; coupled coincidence points; general metric spaces.

\section{INTRODUCTION}

The Banach contraction principle is the most known fixed point theorems. In 1993, Czerwik. ${ }^{9}$ introduced b-metric spaces where the inequality generalized as follows: $\mathrm{d}(\mathrm{x}, \mathrm{z}) \leq \mathrm{b}[\mathrm{d}(\mathrm{x}, \mathrm{y})+\mathrm{d}(\mathrm{y}, \mathrm{z})]$ for all $\mathrm{x}, \mathrm{y}$ and $\mathrm{z} \in \mathrm{X}, \mathrm{b} \geq 1$

In. ${ }^{8}$, Branceciri defined a generalized metric space as a metric space in which the triangle inequality is replaced by the rectangular one called quadrilateral inequality $\mathrm{d}(\mathrm{x}, \mathrm{y}) \leq \mathrm{d}(\mathrm{x}, \mathrm{u})+\mathrm{d}(\mathrm{u}, \mathrm{v})+\mathrm{d}(\mathrm{v}, \mathrm{y})$ for all $\mathrm{x}, \mathrm{y}, \mathrm{u}$ and $\mathrm{v} \in \mathrm{X}$ On the other hand, In. ${ }^{10}$, Dhage introduced the notion of $\mathrm{D}$-metric spaces on $\mathrm{X}^{3}$ :

$$
\begin{aligned}
& \text { 1.D }(x, y, z)=0 \text { if and only if } x=y=z \text { (coincidence). } \\
& \text { 2.D }(x, y, z)=D(p\{x, y, z\}) \text {, for all } x, y, z \in X \text { and for any permutation } p\{x, y, z\} \text { of } x, y, z \text { (symmetry). } \\
& \text { 3.D }(x, y, z) \leq D(x, y, a)+D(x, a, z)+D(a, y, z) \text {, for all } x, y, z \text {, and } a \in X \text { (tetrahedral inequality). }
\end{aligned}
$$

and claimed that D-metric but, Naidu S.V.R., Rao K.P.R. and Rao N.S. (2004-2005) gave many corrections for Dhage's work in. ${ }^{14,15}$ and 16 . In 2006, Mustafa and Sims. ${ }^{25}$ introduce a new concept known as G-metric space satisfied the following:

$$
\begin{aligned}
& \text { 1. } G(x, y, z)=0 \text { iff } x=y=z \text { for all } x, y, z \in X \\
& \text { 2. } G(x, x, y)>0 \text { for all } x, y \in X \text {, with } x \neq y \text {. } \\
& \text { 3. } G(x, x, y) \leq G(x, y, z) \text { for all } x, y, z \in X \text {, with } z \neq y \text {. } \\
& \text { 4. } G(x, y, z)=G(p\{x, y, z\}) \text {, p permutation of } x, y \text { and } z \text {. } \\
& \text { 5. } G(x, y, z) \leq G(x, a, a)+G(a, y, z) \text { for all } x, y, z \text { and } a \in X \text { (Rectangle inequality). }
\end{aligned}
$$

Mustafa et al. studied many fixed point theorems for mappings satisfying several contractive conditions on complete Gmetric space. Aghajani et al. ${ }^{4}$ introduced new generalizations of G-metric spaces called $g_{b}$-metric space. Mustafa et al. ${ }^{13}$ have obtained some coupled coincidence point theorems for $\mathrm{g}_{\mathrm{b}}$-metric space. Kada et al. ${ }^{12}$ introduced the concept of $\mathbf{w}$ - 
distance on a metric space. Saadati et al. ${ }^{17}$ defined an $\rho$-distance on a complete G-metric spaces. Gholizadeh et al. ${ }^{11}$ state complete partially ordered G-metric space with the concept of $\rho$-distance. Shatanawi and Pitea in ${ }^{19,20}$ prove some fixed and coupled fixed point theorem for nonlinear contractions used the notion of $\rho$-distance see ${ }^{1,2,3,5,6,7}$. The aim of this

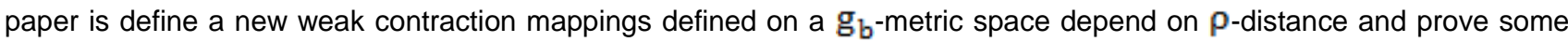
results about the fixed point, coupled coincidence point.

\section{Preliminaries:}

\section{Definition 2-1: ${ }^{13}$}

Let $\mathrm{X}$ be a non-empty set and $\mathrm{Y}: \mathrm{X} \times \mathrm{X} \times \mathrm{X} \rightarrow \mathbb{R}^{+}$be a function such that for all $\mathrm{x}, \mathrm{y}, \mathrm{z}$ and $\mathrm{a} \in \mathrm{X}, \mathrm{b} \geq 1$

1. $\mathrm{y}(\mathrm{x}, \mathrm{y}, \mathrm{z})=0$ if $\mathrm{x}=\mathrm{y}=\mathrm{z}$.

2. $\mathrm{y}(\mathrm{x}, \mathrm{x}, \mathrm{y})>0$ for all $\mathrm{x}, \mathrm{y} \in \mathrm{X}$ with $\mathrm{x} \neq \mathrm{y}$.

3. $\mathrm{y}(\mathrm{x}, \mathrm{x}, \mathrm{y}) \leq \mathrm{y}(\mathrm{x}, \mathrm{y}, \mathrm{z})$ for all $\mathrm{x}, \mathrm{y}, \mathrm{z} \in \mathrm{X}$ with $\mathrm{y} \neq \mathrm{z}$.

4. $y(x, y, z)=y(p\{x, y, z\})$, p permutation of $x, y$ and $z$.

5. $\mathrm{y}(\mathrm{x}, \mathrm{y}, \mathrm{z}) \leq \mathrm{b}[\mathrm{y}(\mathrm{x}, \mathrm{a}, \mathrm{a})+\mathrm{y}(\mathrm{a}, \mathrm{y}, \mathrm{z})]$ for all $\mathrm{x}, \mathrm{y}, \mathrm{z}$ and $\mathrm{a} \in \mathrm{X}, \mathrm{b} \geq 1$ (Like trihedron). then the pair $(\mathrm{X}, \mathrm{Y})$ is called generalized $\mathrm{b}$-metric space.

\section{Definition 2-2: ${ }^{13}$}

Let $\mathrm{X}$ be a $g_{b}-m$ space. A sequence $\left\{x_{n}\right\}$ in $X$ is said to be:

1. $\mathrm{Y}$-Cauchy sequence if, for each $\varepsilon>0$, there is $\mathrm{n}_{0} \in \mathbb{N}$ such that, for all $\mathrm{m}, \mathrm{n}_{,} \mathrm{i} \geq \mathrm{n}_{0}, \mathrm{Y}\left(\mathrm{x}_{\mathrm{n}}, \mathrm{x}_{\mathrm{m}}, \mathrm{x}_{\mathrm{i}}\right)<\varepsilon$.

2. $\mathrm{Y}$-convergent to a point $\mathrm{x} \in \mathrm{X}$ if, for each $\boldsymbol{\varepsilon}>0$, there is $\mathrm{n}_{0} \in \mathbb{N}$ such that, for all $\mathrm{m}, \mathrm{n} \geq \mathrm{n}_{0}, \mathrm{Y}\left(\mathrm{x}_{\mathrm{n}}, \mathrm{x}_{\mathrm{m}}, \mathrm{x}\right)<\varepsilon$. Throughout this paper $(\mathrm{X}, \mathrm{y})$ will be a generalized $\mathrm{b}$-metric space $\mathrm{b} \geq 1$.

\section{Definition 2-3: ${ }^{17}$}

Let $\rho: X \times X \times X \rightarrow \mathbb{R}^{+}, \rho$ is called an $\rho$-distance on $X$ if for all $x_{y}, y, z$ and $a \in X$ :

(a) $\rho(x, y, z) \leq \rho(x, a, a)+\rho(a, y, z)$, for all $x, y, z, a \in X$.

(b) For each $\mathrm{x}, \mathrm{y} \in \mathrm{X}, \rho(\mathrm{x}, \mathrm{y}, \mathrm{.}), \rho(\mathrm{x}, \mathrm{y}): \mathrm{X} \rightarrow \mathbb{R}^{+}$are Lower semi-continuous (L.S.C).

(c) $\forall \varepsilon>0$ there is $\delta>0$ such that $\rho(x, a, a) \leq \delta$ and $\rho(a, y, z) \leq \delta$ imply

$\mathrm{y}(\mathrm{x}, \mathrm{y}, \mathrm{z}) \leq \varepsilon$

Lemma 2-4: 17,11 
Let $\rho$ be an $\rho$-distance on $\mathrm{X}$ and let $\left\{\mathrm{x}_{\mathrm{n}}\right\},\left\{\mathrm{y}_{\mathrm{n}}\right\}$ are sequences in $\mathrm{X},\left\{\alpha_{\mathrm{n}}\right\}$ and $\left\{\beta_{\mathrm{n}}\right\}$ are sequences in $\mathbb{R}^{+}$with $\lim _{\mathrm{n} \rightarrow \infty} \alpha_{\mathrm{n}}=\lim _{\mathrm{n} \rightarrow \infty} \beta_{\mathrm{n}}=0$. If $\mathrm{x}, \mathrm{y}, \mathrm{z}$ and $\mathrm{a} \in \mathrm{X}$ then

(1) If $\rho\left(y_{,} x_{n}, x_{n}\right) \leq \alpha_{n}$ and $\rho\left(x_{n}, y, z\right) \leq \beta_{n}$ for $n \in \mathbb{N}$ then $y(y, y, z)<\varepsilon$ and, $y=z$.

(2) If $\rho\left(y_{n}, x_{n}, x_{n}\right) \leq \alpha_{n}$ and $\rho\left(x_{n}, y_{m}, z\right) \leq \beta_{n}$ for $m>n$ then $y\left(y_{n}, y_{m}, z\right) \rightarrow 0$, hence $y_{n} \rightarrow z$.

(3) If $\rho\left(x_{n}, x_{m}, x_{i}\right) \leq \alpha_{n}$ for $i, n, m \in \mathbb{N}$ with $n \leq m \leq i$, then $\left\{x_{n}\right\}$ is a $y$-Cauchy sequence.

(4) If $\rho\left(x_{n}, a, a\right) \leq \alpha_{n}, n \in \mathbb{N}$ then $\left\{x_{n}\right\}$ is a y-Cauchy sequence.

\section{Definition 2-5: ${ }^{18}$}

Let $\mathrm{G}: \mathrm{X} \times \mathrm{X} \rightarrow \mathrm{X}$ and $\mathrm{T}: \mathrm{X} \rightarrow \mathrm{X}$ be two mapping. An ordered pair $(\mathrm{x}, \mathrm{y}) \in \mathrm{X} \times \mathrm{X}$ is called:

(a) Fixed point if $\mathrm{Tx}=\mathrm{x}$.

(b) Coupled coincidence point if $T(x)=G(x, y)$ and $T(y)=G(y, x)$.

\section{Main Results:}

The following classes are needed in the next results. Let $\mu$ be a class of functions $\mu: \mathbb{R}^{+} \rightarrow \mathbb{R}^{+}$with

i. $\mu$ is continuous.

ii. $\mu$ non-decreasing.

iii. $\mu(\varepsilon)>0$ for all $\varepsilon>0$.

and Let $\Psi$ be a class of functions $\psi: \mathbb{R}^{+} \rightarrow \mathbb{R}^{+}$with

1. $\psi$ non-decreasing.

2. $\psi$ is right continuous.

3. $\psi(\mathrm{t})<0$ for all $\mathrm{t}>0$.

\section{Remark 3-1:}

If $\psi \in \Psi$ then $\lim _{\mathrm{n} \rightarrow \infty} \psi^{\mathrm{n}}(\mathrm{t})=0$ for each $\mathrm{t}>0$ and if $\mu \in \mu,\left\{\mathrm{a}_{\mathrm{n}}\right\} \subseteq \mathbb{R}^{+}$and $\lim _{n \rightarrow \infty} \mu\left(a_{n}\right)=0$ then $\lim _{n \rightarrow \infty} a_{n}=0$

\section{Fixed Point:}

\section{Theorem 3-2:}

Let $\rho$ be an $\rho$-distance, $\mathrm{T}: \mathrm{X} \rightarrow \mathrm{X}$ be a mapping and $\mu \in \boldsymbol{\mu}, \psi \in \Psi$ such that 
Suppose that if $\mathrm{u} \neq \mathrm{Tu}$ then $\inf \{\rho(\mathrm{x}, \mathrm{Tx}, \mathrm{u}): \mathrm{x} \in \mathrm{X}\}>0$

Then $\mathrm{T}$ has a unique fixed point.

\section{Proof:}

Let $\mathrm{x}_{0} \in \mathrm{X}$ and $\mathrm{x}_{\mathrm{n}+1}=\mathrm{Tx}_{\mathrm{n}}, \forall \mathrm{n} \in \mathbb{N}$

if there is $n \in \mathbb{N}$ for which $x_{n+1}=x_{n}$ then $x_{n}$ is fixed point of $T$.

in the following, we assume $\mathrm{x}_{\mathrm{n}+1} \neq \mathrm{x}_{\mathrm{n}}, \forall \mathrm{n} \in \mathbb{N}$

by condition (1)

$\mu \rho\left(\mathrm{x}_{\mathrm{n},} \mathrm{x}_{\mathrm{n}+1}, \mathrm{x}_{\mathrm{n}+1}\right)=\mu \rho\left(\mathrm{Tx}_{\mathrm{n}-1}, \mathrm{Tx}_{\mathrm{n}}, \mathrm{Tx}_{\mathrm{n}}\right)$

$\leq \psi \mu \rho\left(\mathrm{x}_{\mathrm{n}-1}, \mathrm{x}_{\mathrm{n}}, \mathrm{x}_{\mathrm{n}}\right)$

$\vdots$

$\leq \psi^{\mathrm{n}} \mu \rho\left(\mathrm{x}_{0}, \mathrm{x}_{1}, \mathrm{x}_{1}\right)$

thus $\lim _{n \rightarrow \infty} \mu \rho\left(x_{n}, x_{n+1}, x_{n+1}\right)=0$. Then by remark (2-1) implies

$\lim _{n \rightarrow \infty} \rho\left(x_{n}, x_{n+1}, x_{n+1}\right)=0$

also

$\lim _{n \rightarrow \infty} \rho\left(x_{n+1}, x_{n}, x_{n}\right)=0$

Assume that $\left\{\mathrm{x}_{\mathrm{n}}\right\}$ is not a $\mathrm{y}$-Cauchy sequence, so, there is an $\varepsilon>0$ and $\left\{\mathrm{x}_{\mathrm{n}_{\mathrm{k}}}\right\},\left\{\mathrm{x}_{\mathrm{m}_{\mathrm{k}}}\right\}$ subsequences of $\left\{\mathrm{x}_{\mathrm{n}}\right\}$ with $\mathrm{m}_{\mathrm{k}} \geq \mathrm{n}_{\mathrm{k}} \geq \mathrm{k}$ such that

$\rho\left(\mathrm{x}_{\mathrm{n}_{\mathrm{k}}}, \mathrm{x}_{\mathrm{m}_{\mathrm{k}}}, \mathrm{x}_{\mathrm{m}_{\mathrm{k}}}\right) \geq \varepsilon$

$\rho\left(\mathrm{x}_{\mathrm{n}_{\mathrm{k}}}, \mathrm{x}_{\mathrm{m}_{\mathrm{k}}-1}, \mathrm{x}_{\mathrm{m}_{\mathrm{k}}-1}\right)<\varepsilon$

the next step getting from conditions (4) and (5)

$\varepsilon \leq \rho\left(\mathrm{x}_{\mathrm{n}_{\mathrm{k}}}, \mathrm{x}_{\mathrm{m}_{\mathrm{k}}}, \mathrm{x}_{\mathrm{m}_{\mathrm{k}}}\right)$

$\leq \rho\left(x_{n_{k}}, x_{m_{k}-1}, x_{m_{k}-1}\right)+\rho\left(x_{m_{k}-1}, x_{m_{k}}, x_{m_{k}}\right)$

$<\varepsilon+\rho\left(\mathrm{x}_{\mathrm{m}_{\mathrm{k}}-1}, \mathrm{x}_{\mathrm{m}_{\mathrm{k}}}, \mathrm{x}_{\mathrm{m}_{\mathrm{k}}}\right)$

then letting $\mathrm{k} \rightarrow \infty$ in the above inequality and using (2) 
$\lim _{\mathrm{k} \rightarrow \infty} \rho\left(\mathrm{x}_{\mathrm{n}_{\mathrm{k}}}, \mathrm{x}_{\mathrm{m}_{\mathrm{k}}}, \mathrm{x}_{\mathrm{m}_{\mathrm{k}}}\right)=\varepsilon^{+}$

if $\eta=\lim \sup \rho\left(\mathrm{x}_{\mathrm{n}_{\mathrm{k}}+1}, \mathrm{x}_{\mathrm{m}_{\mathrm{k}}+1}, \mathrm{x}_{\mathrm{m}_{\mathrm{k}}+1}\right) \geq \varepsilon$

then there exists $\left\{k_{r}\right\}$ such that

$\rho\left(\mathrm{x}_{\mathrm{n}_{\mathrm{k}}+1}, \mathrm{x}_{\mathrm{m}_{\mathrm{k}_{\mathrm{r}}}+1}, \mathrm{x}_{\mathrm{m}_{\mathrm{k}_{\mathrm{r}}}+1}\right) \rightarrow \mathrm{n} \geq \varepsilon$ as $\mathrm{r} \rightarrow \infty$.

since $\boldsymbol{\mu}$ is continuous and non-decreasing

$\mu(\varepsilon) \leq \mu(\mathrm{r})=\lim _{\mathrm{r} \rightarrow \infty} \mu \rho\left(\mathrm{x}_{\mathrm{n}_{\mathrm{k}_{\mathrm{r}}}+1}, \mathrm{x}_{\mathrm{m}_{\mathrm{k}_{\mathrm{r}}}+1}, \mathrm{x}_{\mathrm{m}_{\mathrm{k}_{\mathrm{r}}}+1}\right)$

$\leq \lim _{\mathrm{r} \rightarrow \infty} \psi \mu \rho\left(\mathrm{x}_{\mathrm{n}_{\mathrm{k}_{\mathrm{r}}},}, \mathrm{x}_{\mathrm{m}_{\mathrm{k}_{\mathrm{r}}}}, \mathrm{x}_{\mathrm{m}_{\mathrm{k}_{\mathrm{r}}}}\right)=\psi \mu(\mathrm{\varepsilon})$

note that $\mu \rho\left(\mathrm{x}_{\mathrm{n}_{\mathrm{k}_{\mathrm{r}}}}, \mathrm{x}_{\mathrm{m}_{\mathrm{k}_{\mathrm{r}}}}, \mathrm{x}_{\mathrm{m}_{\mathrm{k}_{\mathrm{r}}}}\right) \rightarrow \mu(\varepsilon)$, and $\psi$ is right continuous.

thus $\mu(\varepsilon)=0$. This is a contradiction and

$\lim _{\mathrm{k} \rightarrow \infty} \operatorname{supp}\left(\mathrm{x}_{\mathrm{n}_{\mathrm{k}}+1}, \mathrm{x}_{\mathrm{m}_{\mathrm{k}}+1}, \mathrm{x}_{\mathrm{m}_{\mathrm{k}}+1}\right)<\varepsilon$

this implies that

$\varepsilon \leq \rho\left(\mathrm{x}_{\mathrm{n}_{\mathrm{k}}}, \mathrm{x}_{\mathrm{m}_{\mathrm{k}}}, \mathrm{x}_{\mathrm{m}_{\mathrm{k}}}\right)$

$\leq \rho\left(x_{n_{k}}, x_{n_{k}}+1, x_{n_{k}+1}\right)+\rho\left(x_{n_{k}+1}, x_{m_{k}+1}, x_{m_{k}+1}\right)+\rho\left(x_{m_{k}+1}, x_{m_{k}}, x_{m_{k}}\right)$

by (2),(3) and (6)

$\varepsilon \leq \lim _{\mathrm{k} \rightarrow \infty} \mathrm{p}\left(\mathrm{x}_{\mathrm{n}_{\mathrm{k}}}, \mathrm{x}_{\mathrm{n}_{\mathrm{k}}+1}, \mathrm{x}_{\mathrm{n}_{\mathrm{k}}+1}\right)+\lim _{\mathrm{k} \rightarrow \infty} \sup \rho\left(\mathrm{x}_{\mathrm{n}_{\mathrm{k}}+1}, \mathrm{x}_{\mathrm{m}_{\mathrm{k}}+1}, \mathrm{x}_{\mathrm{m}_{\mathrm{k}}+1}\right)$

$+\lim _{\mathrm{k} \rightarrow \infty} \rho\left(\mathrm{x}_{\mathrm{m}_{\mathrm{k}}+1}, \mathrm{x}_{\mathrm{m}_{\mathrm{k}}}, \mathrm{x}_{\mathrm{m}_{\mathrm{k}}}\right)$

$=\lim _{\mathrm{k} \rightarrow \infty} \sup \rho\left(\mathrm{x}_{\mathrm{n}_{\mathrm{k}}+1}, \mathrm{x}_{\mathrm{m}_{\mathrm{k}}+1}, \mathrm{x}_{\mathrm{m}_{\mathrm{k}}+1}\right)<\varepsilon$

a contradiction, then

$\lim _{m, n \rightarrow \infty} \rho\left(x_{n}, x_{m}, x_{m}\right)=0$

then $\left\{\mathrm{x}_{\mathrm{n}}\right\}$ is $\mathrm{y}$-Cauchy sequence. Since $\mathrm{X}$ complete, there exists $\mathrm{u} \in \mathrm{X}$ such that

$\lim _{n \rightarrow \infty} x_{n}=u$

suppose $\mathrm{u} \neq \mathrm{Tu}$

now, for $\varepsilon>0$ and by (L.S.C) of $\rho$, we get 
$\rho\left(x_{n}, x_{m}, u\right) \leq \lim _{p \rightarrow \infty} \inf \rho\left(x_{n}, x_{m}, x_{p}\right) \leq \varepsilon$

considering $\mathrm{m}=\mathrm{n}+1$ in (7), we get

$\rho\left(\mathrm{x}_{\mathrm{n}}, \mathrm{Tx}_{\mathrm{n}}, \mathrm{u}\right) \leq \varepsilon$

on the other hand, we get

$0<\inf \{\rho(x, T x, u): x \in X\}$

$\leq \inf \left\{\rho\left(x_{n}, T_{n}, u\right): n \geq n_{0}\right\} \leq \varepsilon$

this implies that $\inf \{\rho(\mathrm{x}, \mathrm{Tx}, \mathrm{u}): \mathrm{x}, \mathrm{y} \in \mathrm{X}\}=0$

which is contradiction with hypothesis, therefore $\mathrm{u}=\mathrm{Tu}$

Suppose $\mathrm{u}_{1}$ and $\mathrm{u}_{2}$ are two fixed points of $\mathrm{T}_{y}$ we have

$\mu \rho\left(\mathrm{u}_{1}, \mathrm{u}_{2}, \mathrm{u}_{2}\right)=\mu \rho\left(\mathrm{Tu}_{1}, \mathrm{Tu}_{2}, \mathrm{Tu}_{2}\right)$

$\leq \psi \mu \rho\left(\mathrm{u}_{1}, \mathrm{u}_{2}, \mathrm{u}_{2}\right)$

thus, $\mu \rho\left(\mathrm{u}_{1}, \mathrm{u}_{2}, \mathrm{u}_{2}\right)=0$ and $\rho\left(\mathrm{u}_{1}, \mathrm{u}_{2}, \mathrm{u}_{2}\right)=0$

similarly $\rho\left(\mathrm{u}_{1}, \mathrm{u}_{2}, \mathrm{u}_{1}\right)=0$

then, by lemma (2-4) pent (1), we get $\mathrm{u}_{1}=\mathrm{u}_{2}$.

\section{Coupled Coincidence Point:}

\section{Theorem 3-3:}

Let $\rho$ be an $\rho$-distance, $\mathrm{G}: \mathrm{X} \times \mathrm{X} \rightarrow \mathrm{X}$ and $\mathrm{T}: \mathrm{X} \rightarrow \mathrm{X}$ be a mappings with properties $\mathrm{G}(\mathrm{X} \times \mathrm{X}) \subseteq \mathrm{TX}$ and $\mathrm{TX}$ complete subspace of $\mathrm{X}$. Consider $\mu \in \boldsymbol{\mu}, \psi \in \Psi$ such that

$\mu \rho(\mathrm{G}(\mathrm{x}, \mathrm{y}), \mathrm{G}(\mathrm{u}, \mathrm{v}), \mathrm{G}(\mathrm{z}, \mathrm{w})) \leq \psi \mu \rho(\mathrm{Tx}, \mathrm{Tu}, \mathrm{Tz})$ for each $\mathrm{x}, \mathrm{y}, \mathrm{u}, \mathrm{v}, \mathrm{z}, \mathrm{w} \in \mathrm{X}$

If $\mathrm{G}(\mathrm{u}, \mathrm{v}) \neq \mathrm{Tu}$ or $\mathrm{G}(\mathrm{v}, \mathrm{u}) \neq \mathrm{Tv}$ then

$\inf \{\rho(T x, G(x, y), T u)+\rho(T y, G(y, x), T v): x, y \in X\}>0$

Then $\mathrm{G}$ and $\mathrm{T}$ have a unique coupled coincidence point.

\section{Proof:}

Let $\mathrm{x}_{0}, \mathrm{y}_{0} \in \mathrm{X}$, since $\mathrm{G}(\mathrm{X} \times \mathrm{X}) \subseteq \mathrm{TX}$, we can choose $\mathrm{x}_{1}, \mathrm{y}_{1} \in \mathrm{X}$ such that

$\mathrm{Tx}_{1}=\mathrm{G}\left(\mathrm{x}_{0}, \mathrm{y}_{0}\right)$ and $\mathrm{Ty}_{1}=\mathrm{G}\left(\mathrm{y}_{0}, \mathrm{x}_{0}\right)$. Again from $\mathrm{G}(\mathrm{X} \times \mathrm{X}) \subseteq \mathrm{TX}$, we can choose $\mathrm{x}_{2}, \mathrm{y}_{2} \in \mathrm{X}$ such that $\mathrm{Tx}_{2}=\mathrm{G}\left(\mathrm{x}_{1}, \mathrm{y}_{1}\right)$ and $\mathrm{Ty}_{2}=\mathrm{G}\left(\mathrm{y}_{1}, \mathrm{x}_{1}\right)$ 
continuing in the process, we can construct two sequences $\left\{\mathrm{x}_{\mathrm{n}}\right\}$ and $\left\{\mathrm{y}_{\mathrm{n}}\right\}$ in $\mathrm{X}$ such that

$\mathrm{Tx}_{\mathrm{n}+1}=\mathrm{G}\left(\mathrm{x}_{\mathrm{n}}, \mathrm{y}_{\mathrm{n}}\right)$ and $\mathrm{Ty}_{\mathrm{n}+1}=\mathrm{G}\left(\mathrm{y}_{\mathrm{n}}, \mathrm{x}_{\mathrm{n}}\right)$

by (8)

$$
\begin{aligned}
& \mu \rho\left(\mathrm{Tx}_{\mathrm{n}}, \mathrm{Tx}_{\mathrm{n}+1}, \mathrm{Tx}_{\mathrm{n}+1}\right)=\mu \rho\left(\mathrm{G}\left(\mathrm{x}_{\mathrm{n}-1}, \mathrm{y}_{\mathrm{n}-1}\right), \mathrm{G}\left(\mathrm{x}_{\mathrm{n}}, \mathrm{y}_{\mathrm{n}}\right), \mathrm{G}\left(\mathrm{x}_{\mathrm{n}}, \mathrm{y}_{\mathrm{n}}\right)\right) \\
& \leq \psi \mu \rho\left(\mathrm{Tx}_{\mathrm{n}-1}, \mathrm{Tx}_{\mathrm{n}}, \mathrm{Tx}_{\mathrm{n}}\right) \\
& \vdots \\
& \leq \psi^{\mathrm{n}}\left(\mu \rho\left(\mathrm{Tx}_{0}, \mathrm{Tx}_{1}, \mathrm{Tx}_{1}\right)\right.
\end{aligned}
$$$$
\text { then } \lim _{n \rightarrow \infty}\left[\mu \rho\left(\operatorname{Tx}_{n}, \operatorname{Tx}_{n+1}, \operatorname{Tx}_{n+1}\right)\right]=0
$$$$
\text { by remark (2-1) implies }
$$$$
\lim _{n \rightarrow \infty}\left[\rho\left(\mathrm{Tx}_{\mathrm{n}}, \mathrm{Tx}_{\mathrm{n}+1}, \mathrm{Tx}_{\mathrm{n}+1}\right)\right]=0
$$

and

$\lim _{n \rightarrow \infty}\left[\rho\left(\operatorname{Tx}_{n+1}, \operatorname{Tx}_{n}, \mathrm{Tx}_{\mathrm{n}}\right)\right]=0$

also

$\lim _{n \rightarrow \infty}\left[\rho\left(T y_{n}, T y_{n+1}, T y_{n+1}\right)\right]=0$

and

$\lim _{n \rightarrow \infty}\left[\rho\left(T y_{n+1}, T y_{n}, T y_{n}\right)\right]=0$

Assume that at least one of $\left\{\mathrm{Tx}_{\mathrm{n}}\right\}$ or $\left\{\mathrm{Ty}_{\mathrm{n}}\right\}$ is not a $\mathrm{y}$-Cauchy sequence, so, there is an $\varepsilon>0$ and $\left\{\mathrm{Tx}_{\mathrm{n}_{\mathrm{k}}}\right\},\left\{\mathrm{Tx}_{\mathrm{m}_{\mathrm{k}}}\right\}$ subsequences of $\left\{\mathrm{Tx}_{\mathrm{n}}\right\}$ and $\left\{\mathrm{Ty}_{\mathrm{n}_{\mathrm{k}}}\right\},\left\{\mathrm{Ty}_{\mathrm{m}_{\mathrm{k}}}\right\}$

subsequences of $\left\{\mathrm{Ty}_{\mathrm{n}}\right\}$ with $\mathrm{m}_{\mathrm{k}} \geq \mathrm{n}_{\mathrm{k}} \geq \mathrm{k}$ such that

$\rho\left(\mathrm{Tx}_{\mathrm{n}_{\mathrm{k}}}, \mathrm{Tx}_{\mathrm{m}_{\mathrm{k}}}, \mathrm{Tx}_{\mathrm{m}_{\mathrm{k}}}\right) \geq \varepsilon$

$\rho\left(\operatorname{Tx}_{\mathrm{n}_{\mathrm{k}}}, \mathrm{Tx}_{\mathrm{m}_{\mathrm{k}}-1}, \mathrm{Tx}_{\mathrm{m}_{\mathrm{k}}-1}\right)<\varepsilon$

the next step getting from conditions (13) and (14)

$\varepsilon \leq \rho\left(\mathrm{Tx}_{\mathrm{n}_{\mathrm{k}}}, \mathrm{Tx}_{\mathrm{m}_{\mathrm{k}}}, \mathrm{Tx}_{\mathrm{m}_{\mathrm{k}}}\right)$

$\leq \rho\left(\mathrm{Tx}_{\mathrm{n}_{\mathrm{k}}}, \mathrm{Tx}_{\mathrm{m}_{\mathrm{k}}-1}, \mathrm{Tx}_{\mathrm{m}_{\mathrm{k}}-1}\right)+\rho\left(\mathrm{Tx}_{\mathrm{m}_{\mathrm{k}}-1}, \mathrm{Tx}_{\mathrm{m}_{\mathrm{k}}}, \mathrm{Tx}_{\mathrm{m}_{\mathrm{k}}}\right)$

$<\varepsilon+\rho\left(\mathrm{Tx}_{\mathrm{m}_{\mathrm{k}}-1}, \mathrm{Tx}_{\mathrm{m}_{\mathrm{k}}}, \mathrm{Tx}_{\mathrm{m}_{\mathrm{k}}}\right)$

and by (9)as $\mathrm{k} \rightarrow \infty$, 
IS N $2347-1921$

Volume $12 \mathrm{Number} 12$

Jo ur a l of Advancesin Mathematics

$$
\begin{aligned}
& \lim _{\mathrm{k} \rightarrow \infty} \rho\left(\mathrm{Tx}_{\mathrm{n}_{\mathrm{k}}}, \mathrm{Tx}_{\mathrm{m}_{\mathrm{k}}}, \mathrm{Tx}_{\mathrm{m}_{\mathrm{k}}}\right)=\varepsilon^{+} \\
& \text {if } \eta=\lim _{\mathrm{k} \rightarrow \infty} \sup \rho\left(\mathrm{Tx}_{\mathrm{n}_{\mathrm{k}}+1}, \mathrm{Tx}_{\mathrm{m}_{\mathrm{k}}+1}, \mathrm{Tx}_{\mathrm{m}_{\mathrm{k}}+1}\right) \geq \varepsilon
\end{aligned}
$$

then there exists $\left\{\mathrm{k}_{\mathrm{r}}\right\}$ such that

$$
\rho\left(\operatorname{Tx}_{\mathrm{n}_{\mathrm{k}_{\mathrm{r}}}+1}, \mathrm{Tx}_{\mathrm{m}_{\mathrm{k}_{\mathrm{r}}}+1}, \mathrm{Tx}_{\mathrm{m}_{\mathrm{k}_{\mathrm{r}}}+1}\right) \rightarrow \mathrm{\eta} \geq \varepsilon \text { as } \mathrm{r} \rightarrow \infty
$$

since $\mu$ is continuous and non-decreasing

$$
\begin{aligned}
& \mu(\varepsilon) \leq \mu(\mathrm{r})=\lim _{\mathrm{r} \rightarrow \infty} \mu \rho\left(\mathrm{Tx}_{\mathrm{n}_{\mathrm{k}_{\mathrm{r}}}+1}, \mathrm{Tx}_{\mathrm{m}_{\mathrm{k}_{\mathrm{r}}}+1}, \mathrm{Tx}_{\mathrm{m}_{\mathrm{k}_{\mathrm{r}}}+1}\right) \\
& <\lim _{\mathrm{r} \rightarrow \infty} \psi \mu \rho\left(\mathrm{Tx}_{\mathrm{n}_{\mathrm{k}_{\mathrm{r}}}}, \mathrm{Tx}_{\mathrm{m}_{\mathrm{k}_{\mathrm{r}}}}, \mathrm{Tx}_{\mathrm{m}_{\mathrm{k}_{\mathrm{r}}}}\right) \\
& =\psi \mu(\varepsilon) \\
& \text { note that } \mu \rho\left(\mathrm{Tx}_{\mathrm{n}_{\mathrm{k}_{\mathrm{r}}}}, \mathrm{Tx}_{\mathrm{m}_{\mathrm{k}_{\mathrm{r}}}}, \mathrm{Tx}_{\mathrm{m}_{\mathrm{k}_{\mathrm{r}}}}\right) \rightarrow \mu(\varepsilon)
\end{aligned}
$$

and $\psi$ is right continuous. Thus $\mu(\varepsilon)=0$. This is a contradiction and

$$
\lim _{\mathrm{k} \rightarrow \infty} \sup \left(\mathrm{Tx}_{\mathrm{n}_{\mathrm{k}}+1}, \mathrm{Tx}_{\mathrm{m}_{\mathrm{k}}+1}, \mathrm{Tx}_{\mathrm{m}_{\mathrm{k}}+1}\right)<\varepsilon
$$

this implies that

$$
\begin{aligned}
& \varepsilon \leq \rho\left(\operatorname{Tx}_{\mathrm{n}_{k}}, \mathrm{Tx}_{\mathrm{m}_{\mathrm{k}}}, \mathrm{Tx}_{\mathrm{m}_{\mathrm{k}}}\right) \\
& \leq \rho\left(\mathrm{Tx}_{\mathrm{n}_{\mathrm{k}}}, \mathrm{Tx}_{\mathrm{n}_{\mathrm{k}}+1}, \mathrm{Tx}_{\mathrm{n}_{\mathrm{k}}+1}\right)+\rho\left(\mathrm{Tx}_{\mathrm{n}_{\mathrm{k}}+1}, \mathrm{Tx}_{\mathrm{m}_{\mathrm{k}}+1}, \mathrm{Tx}_{\mathrm{m}_{\mathrm{k}}+1}\right)+\rho\left(\mathrm{Tx}_{\mathrm{m}_{\mathrm{k}}+1}, \mathrm{Tx}_{\mathrm{m}_{\mathrm{k}}}, \mathrm{Tx}_{\mathrm{m}_{\mathrm{k}}}\right)
\end{aligned}
$$

by (9),(10) and (15)

$$
\begin{aligned}
& \varepsilon \leq \lim _{\mathrm{k} \rightarrow \infty} \mathrm{p}\left(\mathrm{Tx}_{\mathrm{n}_{\mathrm{k}}}, \mathrm{Tx}_{\mathrm{n}_{\mathrm{k}}+1}, \mathrm{Tx}_{\mathrm{n}_{\mathrm{k}}+1}\right)+\lim _{\mathrm{k} \rightarrow \infty} \sup \rho\left(\mathrm{Tx}_{\mathrm{n}_{\mathrm{k}}+1}, \mathrm{Tx}_{\mathrm{m}_{\mathrm{k}}+1}, \mathrm{Tx}_{\mathrm{m}_{\mathrm{k}}+1}\right) \\
& +\lim _{\mathrm{k} \rightarrow \infty} \rho\left(\mathrm{Tx}_{\mathrm{m}_{\mathrm{k}}+1}, \mathrm{Tx}_{\mathrm{m}_{\mathrm{k}}}, \mathrm{Tx}_{\mathrm{m}_{\mathrm{k}}}\right) \\
& =\lim _{\mathrm{k} \rightarrow \infty} \sup \rho\left(\mathrm{Tx}_{\mathrm{n}_{\mathrm{k}}+1}, \mathrm{Tx}_{\mathrm{m}_{\mathrm{k}}+1}, \mathrm{Tx}_{\mathrm{m}_{\mathrm{k}}+1}\right)<\varepsilon
\end{aligned}
$$

a contradiction, then

$$
\lim _{m, n \rightarrow \infty} \rho\left(\mathrm{Tx}_{n}, \mathrm{Tx}_{\mathrm{m}}, \mathrm{Tx}_{\mathrm{m}}\right)=0
$$

also

$$
\lim _{m, n \rightarrow \infty} \rho\left(T y_{n}, T y_{m}, T y_{m}\right)=0
$$

$6859 \mid \mathrm{P}$ ag e

January 2016

ww w. cirworld.com 
therefore by lemma (1-4) part (3) $\left\{\mathrm{Tx}_{\mathrm{n}}\right\}$ and $\left\{\mathrm{Ty}_{\mathrm{n}}\right\}$ are $\mathrm{y}$-Cauchy sequence, since TX is $\mathrm{y}$-complete, there exists $\mathrm{u}, \mathrm{v} \in \mathrm{X}$ such that

$\lim _{n \rightarrow \infty} \mathrm{Tx}_{\mathrm{n}}=\mathrm{Tu}$ and $\lim _{\mathrm{n} \rightarrow \infty} \mathrm{Ty}_{\mathrm{n}}=\mathrm{Tv}$

suppose $\mathrm{G}(\mathrm{u}, \mathrm{v}) \neq \mathrm{Tu}$ or $\mathrm{G}(\mathrm{v}, \mathrm{u}) \neq \mathrm{Tv}$

Now, for $\varepsilon>0$ and by (L.S.C) of $\rho$, we get

$\rho\left(\mathrm{Tx}_{\mathrm{n}}, \mathrm{Tx}_{\mathrm{m}}, \mathrm{Tu}\right) \leq \lim _{\mathrm{p} \rightarrow \infty} \inf \rho\left(\mathrm{Tx}_{\mathrm{n}}, \mathrm{Tx}_{\mathrm{m}}, \mathrm{Tx}_{\mathrm{p}}\right) \leq \varepsilon$

$\rho\left(\mathrm{Ty}_{n}, \mathrm{Ty}_{\mathrm{m}}, \mathrm{Tv}\right) \leq \lim _{\mathrm{p} \rightarrow \infty} \inf \rho\left(\mathrm{Ty}_{\mathrm{n}}, \mathrm{Ty}_{\mathrm{m}}, \mathrm{Ty}_{\mathrm{p}}\right) \leq \varepsilon$

Considering $\mathrm{m}=\mathrm{n}+1$ in (16) and (17), we get

$\rho\left(\mathrm{Tx}_{\mathrm{n}}, \mathrm{G}\left(\mathrm{x}_{\mathrm{n}}, \mathrm{y}_{\mathrm{n}}\right), \mathrm{Tu}\right)+\rho\left(\mathrm{Ty}_{\mathrm{n}}, \mathrm{G}\left(\mathrm{y}_{\mathrm{n}}, \mathrm{x}_{\mathrm{n}}\right), \mathrm{Tv}\right) \leq 2 \varepsilon$

on the other hand, we get

$0<\inf \{\rho(\mathrm{Tx}, \mathrm{G}(\mathrm{x}, \mathrm{y}), \mathrm{Tu})+\rho(\mathrm{Ty}, \mathrm{G}(\mathrm{y}, \mathrm{x}), \mathrm{Tv}): \mathrm{x}, \mathrm{y} \in \mathrm{X}\}$

$\leq \inf \left\{\rho\left(\mathrm{Tx}_{n}, \mathrm{G}\left(\mathrm{x}_{\mathrm{n}}, \mathrm{y}_{\mathrm{n}}\right), \mathrm{Tu}\right)+\rho\left(\mathrm{Ty}_{\mathrm{n}}, \mathrm{G}\left(\mathrm{y}_{\mathrm{n}}, \mathrm{x}_{\mathrm{n}}\right), \mathrm{Tv}\right): \mathrm{n} \geq \mathrm{n}_{0}\right\} \leq 2 \varepsilon$

this implies that $\inf \{\rho(\mathrm{Tx}, \mathrm{G}(\mathrm{x}, \mathrm{y}), \mathrm{Tu})+\rho(\mathrm{Ty}, \mathrm{G}(\mathrm{y}, \mathrm{x}), \mathrm{Tv}): \mathrm{x}, \mathrm{y} \in \mathrm{X}\}=0$

which is contradiction with hypothesis, therefore $\mathrm{G}(\mathrm{u}, \mathrm{v})=\mathrm{Tu}$ and $\mathrm{G}(\mathrm{v}, \mathrm{u})=\mathrm{Tv}$

Now we prove the uniqueness

assume that $(\mathrm{u}, \mathrm{v})$ and $\left(\mathrm{u}^{*}, \mathrm{v}^{*}\right)$ be a another coupled coincidence point of $\mathrm{G}$ and $\mathrm{T}$

by (8)

$\mu \rho\left(\mathrm{Tu}^{*}, \mathrm{Tu}, \mathrm{Tu}\right)=\mu \rho\left(\mathrm{G}\left(\mathrm{u}^{*}, \mathrm{v}^{*}\right), \mathrm{G}(\mathrm{u}, \mathrm{v}), \mathrm{G}(\mathrm{u}, \mathrm{v})\right)$

$\leq \psi \mu \rho\left(\mathrm{Tu}^{*}, \mathrm{Tu}, \mathrm{Tu}\right)$

then $\mu \rho\left(\mathrm{Tu}^{*}, \mathrm{Tu}, \mathrm{Tu}\right)=0$ then $\rho\left(\mathrm{Tu}^{*}, \mathrm{Tu}, \mathrm{Tu}\right)=0$

similarly $\rho\left(\mathrm{Tu}, \mathrm{Tu}^{*}, \mathrm{Tu}\right)=0$

then by lemma (2-4) pent (1), then $\mathrm{Tu}=\mathrm{Tu}^{*}$

similarly we can show that $\mathrm{Tv}=\mathrm{Tv}^{*}$.

now, by (3.8)

$\mu \rho(\mathrm{Tu}, \mathrm{Tu}, \mathrm{Tv})=\mu \rho(\mathrm{G}(\mathrm{u}, \mathrm{v}), \mathrm{G}(\mathrm{u}, \mathrm{v}), \mathrm{G}(\mathrm{v}, \mathrm{u}))$ 
$\leq \psi \mu \rho(\mathrm{Tu}, \mathrm{Tu}, \mathrm{Tv})$

then $\mu \rho(\mathrm{Tu}, \mathrm{Tu}, \mathrm{Tv})=0$ then $\rho(\mathrm{Tu}, \mathrm{Tu}, \mathrm{Tv})=0$

also $\rho(\mathrm{Tu}, \mathrm{Tv}, \mathrm{Tu})=0$

then, by lemma (2-4) pent (1), then $\mathrm{Tu}=\mathrm{Tv}$.

The following example illustrate theorem (2-2)

\section{Example 3-4:}

Consider $(\mathrm{X}, \mathrm{y}) \mathrm{g}_{\mathrm{b}}-\mathrm{m}$ space with $\mathrm{b}=1$ define as follows

$\mathrm{X}=\{0,1,2, \ldots\}$ define $\mathrm{y}: \mathrm{X} \times \mathrm{X} \times \mathrm{X} \rightarrow \mathbb{R}^{+}$by

$y(x, y, z)= \begin{cases}0 & \text { if } x=y=z \\ x+y+z & \text { if } x \neq y \text { or } y \neq z \text { or } x \neq z\end{cases}$

-distance, $\rho: X \times X \times X \rightarrow X, \rho(x, y, z)=x+2 \max \{y, z\} \rho$ is $\rho$

Define $\mathrm{T}: \mathrm{X} \rightarrow \mathrm{X}$

$\mathrm{Tx}= \begin{cases}0 & \text { if } \mathrm{x}=0,1 \\ \mathrm{x}-1 & \text { if } \mathrm{x} \geq 2\end{cases}$

and $\mu: \mathbb{R}^{+} \rightarrow \mathbb{R}^{+}, \mu(\mathrm{t})=4 \mathrm{t}, \psi: \mathbb{R}^{+} \rightarrow \mathbb{R}^{+}, \psi(\mathrm{t})=\mathrm{t}, \mathrm{t}>0$

If $\mathrm{u} \neq \mathrm{Tu}$ then

$\inf \{\rho(x, T x, u): x \in X\} \geq \inf \{x+2 u: x \in X\} \geq 2 u>0$

for $\mathrm{x}, \mathrm{y}, \mathrm{z} \in \mathrm{X}$, with $\mathrm{y} \geq \mathrm{z}$, then

$\rho(x, y, z)=x+2 y$ and $\rho(T x, T y, T z)=x-1+2(y-1)$

Since

$4[x-1+2(y-1)] \leq 4[x+2 y]$

We have

$\mu \rho(\mathrm{Tx}, \mathrm{Ty}, \mathrm{Tz}) \leq \psi \mu \rho(\mathrm{x}, \mathrm{y}, \mathrm{z})$

thus all hypotheses of theorem (3-2) are satisfied and $\mathrm{x}=0$ is the unique fixed point of $\mathrm{T}$.

\section{REFERENCES}

1. Abbas, M., Ali Khan, M. and Radenovic, S. 2010. Common Coupled Fixed Point Theorems in Cone Metric Spaces for w-Compatible Mappings, Appl. Math. Comput. 217, 195-202. 
2. Abed, S.S. and Jabbar, H.A. 2016. Coupled Points for Total Weakly Contraction Mappings Via $g_{b}-m$ Space, inter. J. of advan. Scie. and tech. resear., Issur 6, vol.3, pages 64-79.

3. Abed, S.S. and Jabbar, H.A. 2016. Coupled Points for Total Weakly Contraction Mappings Via $\rho$-distance, Inter. J. of Basic and Applied Sciences, Vol. 5, No. 3.

4. Aghajani, A., Abbas, M. and Roshan, J.R. 2014. Common Fixed Point of Generalized Weak Contractive Mappings in Partially Ordered $\mathrm{G}_{\mathrm{b}}$-Metric Spaces, Math. Slovaca, in press, Vo.64, pp. 941-960.

5. Agarwal, R.P, Sintunavarat ,W. and Kumam, P. 2013. Coupled Coincidence Point and Common Coupled Fixed Point Theorems Lacking The Mixed Monotone Property, Fixed Point Theory Appl. 2013, Article ID 22.

6. Aydi, H., Postolache, M. and Shatanawi, W. 2012. Coupled Fixed Point Results For $(\psi, \phi)$-Weakly Contractive Mappings in Ordered G-Metric Spaces, Comput. Math. Appl. 63, 298-309.

7. Berinde, V. 2012. Coupled Fixed Point Theorems for Contractive Mixed Monotone Mappings in Partially Ordered Metric Spaces, Nonlinear Anal. 75, 3218-3228.

8. Branciari, A. 2000. A Fixed Point Theorem of Banach-Caccioppoli Type on a Class of Generalized Metric Spaces, publ. Math. Debrcen , 57:1-2, 31-37.

9. Czerwik, S. 1993. Contraction Mappings in b-Metric Spaces, Acta Math. Inform. Univ. Ostrav. 1,5-11.

10. Dhage, B.C. 1992. Generalized Metric Spaces and Mappings With Fixed Points, Bull.Cal.Math.Soc., Vol. 84, pp.(329336).

11. Gholizadeh, L., Saadati, R., Shatanawi, W. and Vaezpour, S.M. 2011. Contractive Mapping in Generalized Ordered Metric Spaces With Application in Integral Equations, Math. Probl. Eng. 2011, Article ID 380784, 14 pages.

12. Kada, O., Suzuki, T. and Takahashi, W. 1996. Non-Convex Minimization Theorems and Fixed Point Theorems in Complete Metric Spaces, Mathematica Japonica, Vol. 44, No. 2, pp. 381-391.

13. Mustafa, Z., Roshan, J.R. and Parvaneh, V. 2013. Coupled Coincidence Point Results for $(\psi, \varphi)$-Weakly Contractive Mappings in Partially Ordered $\mathrm{G}_{\mathrm{b}}$-Metric Spaces, Fixed Point Theory Appl. ,206.

14. Naidu, S.V.R., Rao, K.P.R. and Rao, N.S. 2004. on The Topology of D-Metric Spaces and Generation of D-Metric Spaces From Metric Spaces, Int. J. Math. Sci., No.51, PP. 2719-2740.

15. Naidu, S.V.R., Rao, K.P.R. and Rao, N.S. 2005. on The Concepts of Balls in D-Metric Space, International Journal of Mathematics and Mathematical Sciences, Vol. 1, pp. 133-141.

16. Naidu, S.V.R., Rao, K.P.R. and Rao, N.S. 2005. on Convergent Sequences and Fixed Point Theorems in D-Metric Spaces, International Journal of Mathematics and Mathematical Sciences, Vol.12, pp. 1969-1988.

17. Saadati, R., Vaezpour, S.M., Vetro, P. and Rhoades, B.E. 2010. Fixed Point Theorems in Generalized Partially Ordered G-Metric Spaces, Math. Comput. Modelling. 52,797-801.

18. Sabetghadam, F., Masiha, H.P. and Sanatpour, A.H. 2009. Some Coupled Fixed Point Theorems in Cone Metric Spaces, Fixed point Theory and Applications, Vol. 2009, Article ID 125426, 8 pages.

19. Shatanawi, W. and Pitea, A. 2013. Fixed and Coupled Fixed Point Theorems of Omega-Distance for Nonlinear Contraction, Fixed Point Theory Appl., 2013, 16 pages.

20. Shatanawi, W. and Pitea, A. 2013. Omega-Distance and Coupled Fixed Point in G-Metric Spaces, Fixed Point Theory Appl., 2013, 15 pages. 